\title{
DNA methylation profiling identifies potentially significant epigenetically-regulated genes in glioblastoma multiforme
}

\author{
SHIFENG KAN*, SONG CHAI*, WENHUA CHEN and BO YU \\ Department of Rehabilitation, Shanghai General Hospital, Shanghai Jiaotong University, Shanghai 200080, P.R. China
}

Received April 2, 2018; Accepted April 5, 2019

DOI: $10.3892 / \mathrm{ol} .2019 .10512$

\begin{abstract}
Glioblastoma multiforme (GBM) is one of the most lethal and damaging types of human cancer. The current study was conducted to identify differentially methylated genes (DMGs) between GBM and normal controls, and to improve our understanding of GBM at the epigenetic level. The DNA methylation profile of GBM was downloaded from the Gene Expression Omnibus (GEO) database using the accession number GSE50923. The MethyAnalysis package was applied to identify DMGs between GBM and controls, which were then analyzed by functional enrichment analysis. Protein-protein interaction (PPI) networks were constructed using the hypermethylated and hypomethylated genes. Finally, transcription factors (TFs) that can regulate the hypermethylated and hypomethylated genes were predicted, followed by construction of transcriptional regulatory networks. Furthermore, another relevant dataset, GSE22867, was downloaded from the GEO database for data validation. A total of 476 hypermethylated and 850 hypomethylated genes were identified, which were mainly associated with the functions of 'G-protein-coupled receptors ligand binding', 'cytokine activity', 'cytokine-cytokine receptor interaction', and 'D-glutamine and D-glutamate metabolism'. The hypermethylated gene neuropeptide Y $(N P Y)$ and the hypomethylated gene tumor necrosis factor $(T N F)$ demonstrated high degrees in the PPI network. Forkhead box protein A1 (FOXA1), potassium voltage-gated channel subfamily $\mathrm{C}$ member $3(K C N C 3)$ and caspase-8 (CASP8) exhibited high degrees in the transcriptional regulatory networks. In addition, the methylation profiles of $N P Y, T N F, F O X A 1, K C N C 3$ and $C A S P 8$ were confirmed by another dataset. In summary, the
\end{abstract}

Correspondence to: Dr Bo Yu, Department of Rehabilitation, Shanghai General Hospital, Shanghai Jiaotong University, 100 Haining Road, Shanghai 200080, P.R. China

E-mail: daodu042shizhao@163.com

*Contributed equally

Key words: glioblastoma multiforme, abnormal methylation, transcription factors, functional enrichment analysis, protein-protein interaction network present study systematically analyzed the DNA methylation profile of GBM using bioinformatics approaches and identified several abnormally methylated genes, providing insight into the molecular mechanism underlying GBM.

\section{Introduction}

Glioblastoma multiforme (GBM), which develops from astrocytes, is the most common primary brain tumor in adults and one of the most damaging types of human cancer. Despite aggressive multimodal treatment with surgery, radiotherapy and chemotherapy, the prognosis of GBM is extremely poor $(1,2)$. Additionally, GBM demonstrates a high potential to infiltrate the brain parenchyma, which poses a challenge to the available treatment strategies. Typically, GBM results in mortality at 12-15 months post-diagnosis (1). Therefore, novel therapies for GBM are urgently required.

Identifying the mechanisms underlying the development of GBM is important for the development of new treatments. The genetic alterations that affect genes controlling cell growth, apoptosis and invasion have been widely examined in GBM (3). Epigenetic alterations have also been identified to be involved in GBM by affecting the expression of cancer-associated genes alone or in combination with genetic mechanisms (4). Aberrant methylation of gene promoters is the most widely studied epigenetic change that occurs during oncogenesis. It is understood that increased methylation, termed hypermethylation, in the $\mathrm{CpG}$ island promotes carcinogenesis by silencing tumor suppressor genes, while loss of methylation, termed hypomethylation, enhances the transcriptional activation of oncogenes and induces chromosomal instability $(5,6)$. Decreased expression of tumor suppressor genes, including retinoblastoma gene, phosphatase and tensin homolog, and TP53, is associated with $\mathrm{CpG}$ island promoter hypermethylation and has been reported in GBM (7-9). Epigenetic silencing of O6-methylguanine-DNA methyltransferase, a DNA repair gene that can protect cancer cells from chemotherapeutic alkylating agents, has been revealed to be significantly associated with longer survival times in patients with GBM who are treated with alkylating agents $(10,11)$. Such epigenetic changes may be promising targets for epigenetic anticancer treatments. Indeed, the DNA-demethylating agents 5-azacitidine and 5-aza-2'-deoxycitidine have been approved by the Food and Drug Administration for the treatment of myelogenous leukemia and myelodysplastic syndromes (12). 
Bioinformatics tools and algorithms assist the processing and analysis of high-throughput DNA methylation data $(13,14)$. For example, a previous study used a joint analysis of DNA methylation and gene expression data of GBM to demonstrate that changes in DNA methylation can be associated with survival outcome (15). In addition, a recent study used a computational approach to integrate gene expression and genomic or methylation data to investigate biological networks in GBM (16). The current study used bioinformatics approaches to reanalyze the DNA methylation data deposited by Lai et al (17). The present results may improve understanding of the epigenetic regulation mechanism of GBM and provide potential gene methylation biomarkers for GBM, which may contribute to the development of treatments.

\section{Materials and methods}

Microarray data. DNA methylation data from the GBM study by Lai et al (17) were retrieved from the National Center for Biotechnology Information Gene Expression Omnibus (GEO) database (http://www.ncbi.nlm.nih.gov/projects/geo/) with the accession number GSE50923. The DNA methylation profiles of 54 GBM samples and 24 control brain samples were previously investigated using Illumina HumanMethylation27 BeadChip. The patient details are presented in the original study by Lai et al (17). Only data from 31 GBM and 24 control brain samples were present in the GEO database, therefore, only these data were analyzed in the current study.

Identification of differentially methylated regions (DMRs). The original methylation data were processed using the Bioconductor lumi package version 2.18.0, which is designed to process the Illumina microarray data $(18,19)$. Following quality control and background correction, these data were scaled by quantile normalization as implemented in the lumi package. The methyAnalysis package version 1.8.0 (20) was applied to identify DMRs between GBM and controls. $\mathrm{P}<0.001$ and a difference in methylation levels, calculated as the M-value (19), of $>1$ or $<-1$ were considered to indicate a statistically significant DMR. The identified DMRs were then annotated using the methyAnalysis package and DMRs located at gene promoter regions, within $2 \mathrm{~kb}$ upstream of the transcription start site, were selected for further analysis.

Functional enrichment analysis. Functional enrichment analysis was conducted to explore the most significant differentially methylated genes (DMGs) with relevant biological functions. Gene Ontology (GO) is a bioinformatics tool for annotating genes, gene products and sequences using defined GO terms (21). The Kyoto Encyclopedia of Genes and Genomes (KEGG) is a comprehensive database linking genomic data, stored in the GENES database, with higher order functional information, stored in the PATHWAY database (22). Reactome is a free online database of biological pathways (23). The KEGG pathway, Reactome pathway and GO functional terms for the DMGs were identified using the following cut-off criteria: $\mathrm{P}<0.05$ and number of over-represented genes $>2$.

Construction of a protein-protein interaction (PPI) network. The PPI data were retrieved from the STRING database (http://string.embl.de/). Subsequently, the DMGs were mapped into these interactions, and DMG pairs with an interaction score $>0.9$ were selected to construct the PPI networks, which were visualized using Cytoscape software version 3.1.0 (24).

Classification of DMGs. TSGene (25) is a database for tumor suppressor genes (TSGs) and the TAG database (26) provides information on well-characterized oncogenes and TSGs. The known TSGs and oncogenes were identified from the list of DMGs based on information retrieved from the TSGene and TAG databases.

Identification of transcription factors (TFs) regulating DMGs and construction of a transcriptional regulatory network. The TF regulation data were downloaded from the Encyclopedia of DNA Elements data portal $(27,28)$, and TFs that regulate the hypermethylated genes and hypomethylated genes were identified. The hypergeometric distribution was used to assign a P-value for the prediction of these TFs and an adjusted P-value of $<0.05$ was considered significant. Furthermore, transcriptional regulatory networks of hypermethylated genes and hypomethylated genes were constructed using Cytoscape (version 3.6.0) (24).

Data validation. To validate the identified DMGs from GSE50923, another relevant DNA Methylation Profiling dataset, GSE22867 (29), was downloaded from the GEO database and used for data validation. GSE22867 included 55 GBM samples and 3 control brain samples. The platform was GPL8490 Illumina HumanMethylation27 BeadChip (HumanMethylation27_270596_v.1.2). The $\beta$-value was calculated based on the methylated and unmethylated signal of the sample data, and a t-test was implemented using the genefilter package (version 1.56.0) (30) to identify significant differentially methylated $\mathrm{CpGs}(\mathrm{P}<0.05)$. In addition, selected $\mathrm{CpG}$ sites exhibited a mean methylation ( $\beta$-value) difference $\geq 0.05$ between the disease group and the control group. Subsequently, the genes covering the differentially methylated CpGs were identified and compared with the identified DMGs from GSE50923.

\section{Results}

Identification of DMGs. Based on the methyAnalysis package, a total of 2,407 DMRs were identified between the GBM samples and normal controls. Among these DMRs, 476 hypermethylated and 850 hypomethylated regions were located in gene promoters, which clearly distinguished GBM from normal samples (Fig. 1).

Functional enrichment analysis. To gain insight into the dysregulated biological processes induced by DMGs, separate functional enrichment analyses were performed for the hypermethylated and hypomethylated genes. The data indicated that certain KEGG pathways, including 'neuroactive ligand-receptor interaction', and 'D-glutamine and D-glutamate metabolism', were enriched by hypermethylated genes, while 'cytokine-cytokine receptor interaction' and 'hematopoietic cell lineage' were enriched by hypomethylated genes (Table I). GO terms were grouped into the following three categories: 
Table I. Top five KEGG pathways enriched by hypermethylated genes and hypomethylated genes.

A, Hypermethylated genes

\begin{tabular}{lll}
\hline KEGG ID & \multicolumn{1}{c}{ KEGG pathway } & \multicolumn{1}{c}{ P-value } \\
\hline 4080 & Neuroactive ligand-receptor interaction & $1.18 \times 10^{-3}$ \\
471 & D-Glutamine and D-glutamate metabolism & $3.62 \times 10^{-3}$ \\
4114 & Oocyte meiosis & $6.76 \times 10^{-3}$ \\
250 & Alanine, aspartate and glutamate metabolism & $7.87 \times 10^{-3}$ \\
4971 & Gastric acid secretion & $1.02 \times 10^{-2}$ \\
\hline
\end{tabular}

B, Hypomethylated genes

\begin{tabular}{llr}
\hline KEGG ID & \multicolumn{1}{c}{ KEGG pathway } & P-value \\
\hline 4060 & Cytokine-cytokine receptor interaction & $1.22 \times 10^{-10}$ \\
4640 & Hematopoietic cell lineage & $1.49 \times 10^{-5}$ \\
5150 & Staphylococcus aureus infection & $1.35 \times 10^{-4}$ \\
4610 & Complement and coagulation cascades & $2.72 \times 10^{-4}$ \\
\hline
\end{tabular}

KEGG, Kyoto Encyclopedia of Genes and Genomes.

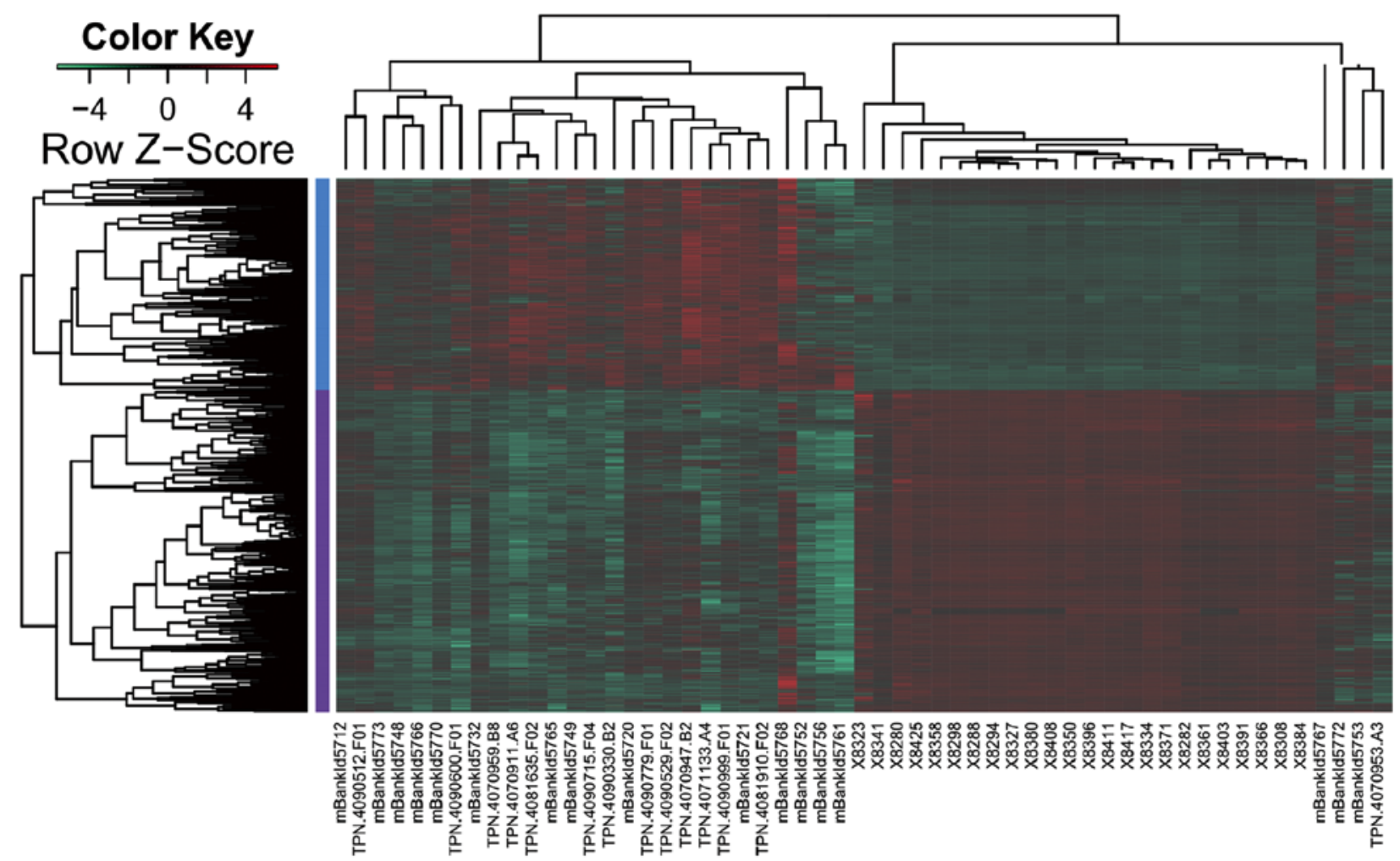

Figure 1. Cluster analysis of differentially methylated genes between patients with glioblastoma multiforme and controls. Each row represents a single gene and each column represents a single sample. Red represents hypermethylated genes and green indicates hypomethylated genes.

Biological process, molecular function and cellular component. The most significantly enriched GO biological process and molecular function terms were 'anatomical structure development' and 'sequence-specific DNA-binding transcription factor activity', respectively, while no statistically significant enriched cellular component terms were identified (Table II).
For hypomethylated genes, the most significant GO terms were 'defense response', 'cytokine activity' and 'extracellular region' (Table II). Reactome pathway analysis demonstrated that both hypermethylated and hypomethylated genes were closely associated with the 'G-protein-coupled receptor (GPCR) ligand binding pathway' (Table III). 
Table II. Top five GO terms enriched by hypermethylated genes and hypomethylated genes.

A, Hypermethylated genes

\begin{tabular}{lll}
\hline GO ID & & \multicolumn{1}{c}{ GO term } \\
\hline $\begin{array}{l}\text { Biological process } \\
\text { GO:0048856 }\end{array}$ & Anatomical structure development & $3.19 \times 10^{-8}$ \\
GO:0032502 & Developmental process & $4.06 \times 10^{-8}$ \\
GO:0044707 & Single-multicellular organism process & $4.85 \times 10^{-8}$ \\
GO:0032501 & Multicellular organismal process & $8.26 \times 10^{-8}$ \\
GO:0007275 & Multicellular organismal development & $3.47 \times 10^{-7}$ \\
Molecular function & & $5.04 \times 10^{-9}$ \\
GO:0003700 & Sequence-specific DNA binding transcription factor activity & $5.51 \times 10^{-9}$ \\
GO:0001071 & Nucleic acid binding transcription factor activity & $1.76 \times 10^{-8}$ \\
GO:0043565 & Sequence-specific DNA binding & $6.04 \times 10^{-7}$ \\
GO:0044212 & Transcription regulatory region DNA binding & $9.99 \times 10^{-7}$ \\
GO:0000975 & Regulatory region DNA binding & \\
Cellular component & & $2.37 \times 10^{-1}$ \\
GO:0044459 & Plasma membrane part & $2.61 \times 10^{-1}$ \\
GO:0019897 & Extrinsic to plasma membrane \\
GO:0008076 & Voltage-gated potassium channel complex \\
GO:0034705 & Potassium channel complex \\
GO:0019898 & Extrinsic to membrane & $4.53 \times 10^{-1}$ \\
\hline
\end{tabular}

B, Hypomethylated genes

\begin{tabular}{llr}
\hline GO ID & & GO term \\
\hline Biological process & & P-value \\
GO:0006952 & Defense response & $8.19 \times 10^{-12}$ \\
GO:0006954 & Inflammatory response & $9.55 \times 10^{-12}$ \\
GO:0050896 & Response to stimulus & $6.68 \times 10^{-11}$ \\
GO:0002376 & Immune system process & $8.73 \times 10^{-11}$ \\
GO:0009611 & Response to wounding & $6.82 \times 10^{-10}$ \\
Molecular function & & $3.13 \times 10^{-7}$ \\
GO:0005125 & Cytokine activity & $1.30 \times 10^{-5}$ \\
GO:0005126 & Cytokine receptor binding & $6.25 \times 10^{-5}$ \\
GO:0004872 & Receptor activity & $1.39 \times 10^{-3}$ \\
GO:0005102 & Receptor binding & $8.86 \times 10^{-3}$ \\
GO:0005506 & Iron ion binding & $<1.00 \times 10^{-3}$ \\
Cellular component & & $<1.00 \times 10^{-3}$ \\
GO:0005576 & Extracellular region & $<1.00 \times 10^{-3}$ \\
GO:0005615 & Extracellular space & $3.84 \times 10^{-9}$ \\
GO:0044421 & Extracellular region part & $5.56 \times 10^{-9}$ \\
GO:0071944 & Cell periphery & \\
GO:0005886 & Plasma membrane &
\end{tabular}

Construction of PPI networks. A total of 476 hypermethylated and 850 hypomethylated genes were mapped to the STRING database and significant interactions with scores $>0.9$ were selected. A total of 83 hypermethylated genes and 204 hypomethylated genes were screened to separately construct PPI networks involving hypermethylated genes (Fig. 2) and hypomethylated genes (Fig. 3). The DMGs adenylate cyclase type 2 ( $A D C Y 2$; degree, 18), neuropeptide Y (NPY; degree, 11) and somatostatin (SST; degree, 11) demonstrated the highest degrees, determined by the number of interactions in the hypermethylated gene network. Kininogen 1 (KNG1; degree, 27), proto-oncogene tyrosine protein kinase $(S R C$; degree, 18$)$ and 
Table III. Top five Reactome pathways enriched by hypermethylated genes and hypomethylated genes.

A, Hypermethylated genes

\begin{tabular}{llr}
\hline Reactome ID & \multicolumn{1}{c}{ Name } & P-value \\
\hline 500792 & GPCR ligand binding & $8.88 \times 10^{-2}$ \\
112316 & Neuronal system & $1.61 \times 10^{-1}$ \\
373076 & Class A/1 (Rhodopsin-like receptors) & $3.55 \times 10^{-1}$ \\
418597 & G $\alpha(z)$ signaling events & $4.98 \times 10^{-1}$ \\
1296072 & Voltage-gated potassium channels & $9.92 \times 10^{-1}$ \\
\hline
\end{tabular}

B, Hypomethylated genes

\begin{tabular}{lll}
\hline Reactome ID & \multicolumn{1}{c}{ Name } & P-value \\
\hline 500792 & GPCR ligand binding & $5.94 \times 10^{-4}$ \\
211897 & Cytochrome P450-arranged by substrate type & $1.05 \times 10^{-3}$ \\
373076 & Class A/1 (Rhodopsin-like receptors) & $5.68 \times 10^{-3}$ \\
211945 & Phase 1-Functionalization of compounds & $6.96 \times 10^{-3}$ \\
\hline
\end{tabular}

GPCR, G-protein-coupled receptor.

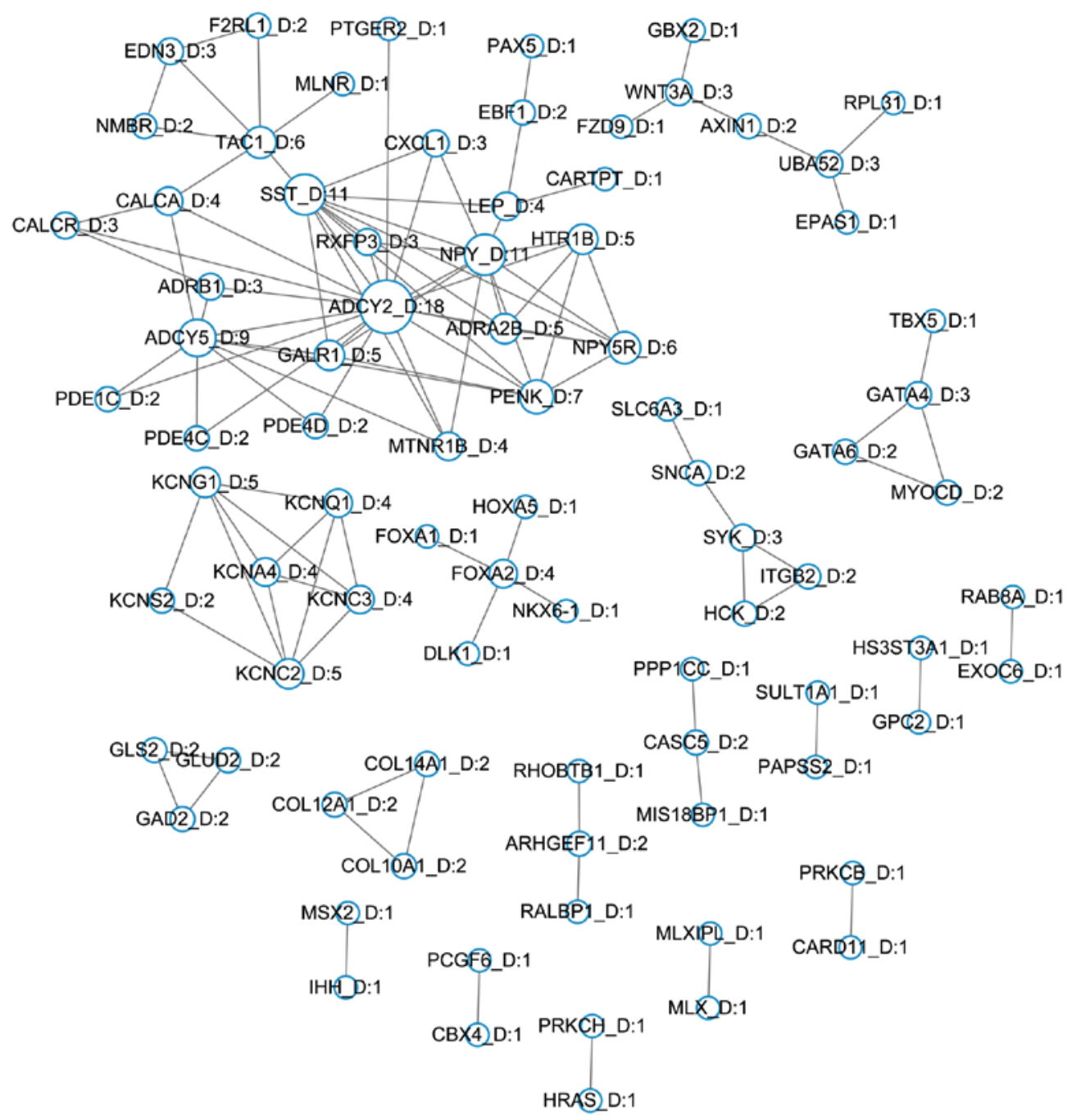

Figure 2. Protein-protein interaction networks for hypermethylated genes. White nodes with blue circles represent the hypermethylated genes and the node size indicates the degree value (larger nodes possess a higher degree). The degree display format of the node is gene_D: num, where 'gene' indicates the gene name, ' $\mathrm{D}$ ' stands for degree and 'num' is the degree value. Lines/edges represent interactions. 


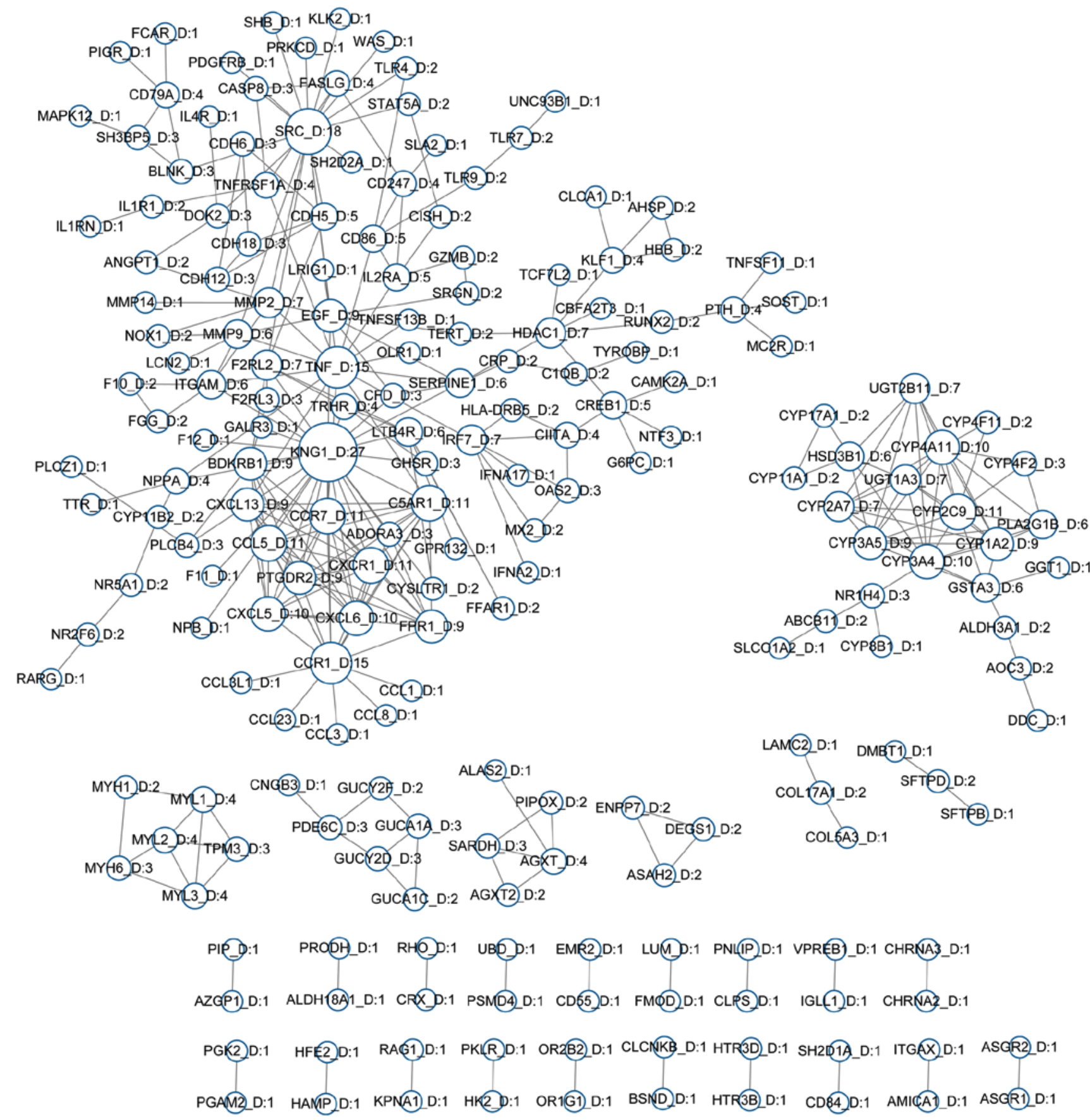

Figure 3. Protein-protein interaction network for hypomethylated genes. White nodes with blue circles represent the hypermethylated genes and the node size indicates the degree value (larger nodes possess a higher degree). The degree display format of the node is gene_D: num, where 'gene' indicates the gene name, ' $D$ ' stands for degree and 'num' is the degree value. Lines/edges represent interactions.

tumor necrosis factor (TNF; degree, 15) exhibited the highest degrees in the hypomethylated gene network.

Classification of the DMGs. A total of 43 cancer-associated genes were identified among the hypermethylated genes, including 8 oncogenes and 25 TSGs, while 41 hypomethylated genes were revealed to be cancer-associated genes, including 10 oncogenes and 24 TSGs.

Prediction of TFs that target the DMGs and construction of transcriptional regulatory networks. A total of 55 TFs that could regulate the hypermethylated genes were identified, including polycomb repressive complex 2 subunit, neuron-restrictive silencer factor and cohesin complex component. Additionally, $55 \mathrm{TFs}$ that could regulate the hypomethylated genes were identified, including signal transducer and activator of transcription 3, and forkhead box protein A1 (FOXAl). Notably, FOXAl was also revealed to be hypermethylated. The transcriptional regulatory networks of hypermethylated genes and hypomethylated genes are presented in Figs. 4 and 5, respectively. The top 10 hypermethylated genes and hypomethylated genes with high node degrees in the regulatory networks are listed in Table IV, including the hypermethylated genes, FOXA1 (degree, 35), 


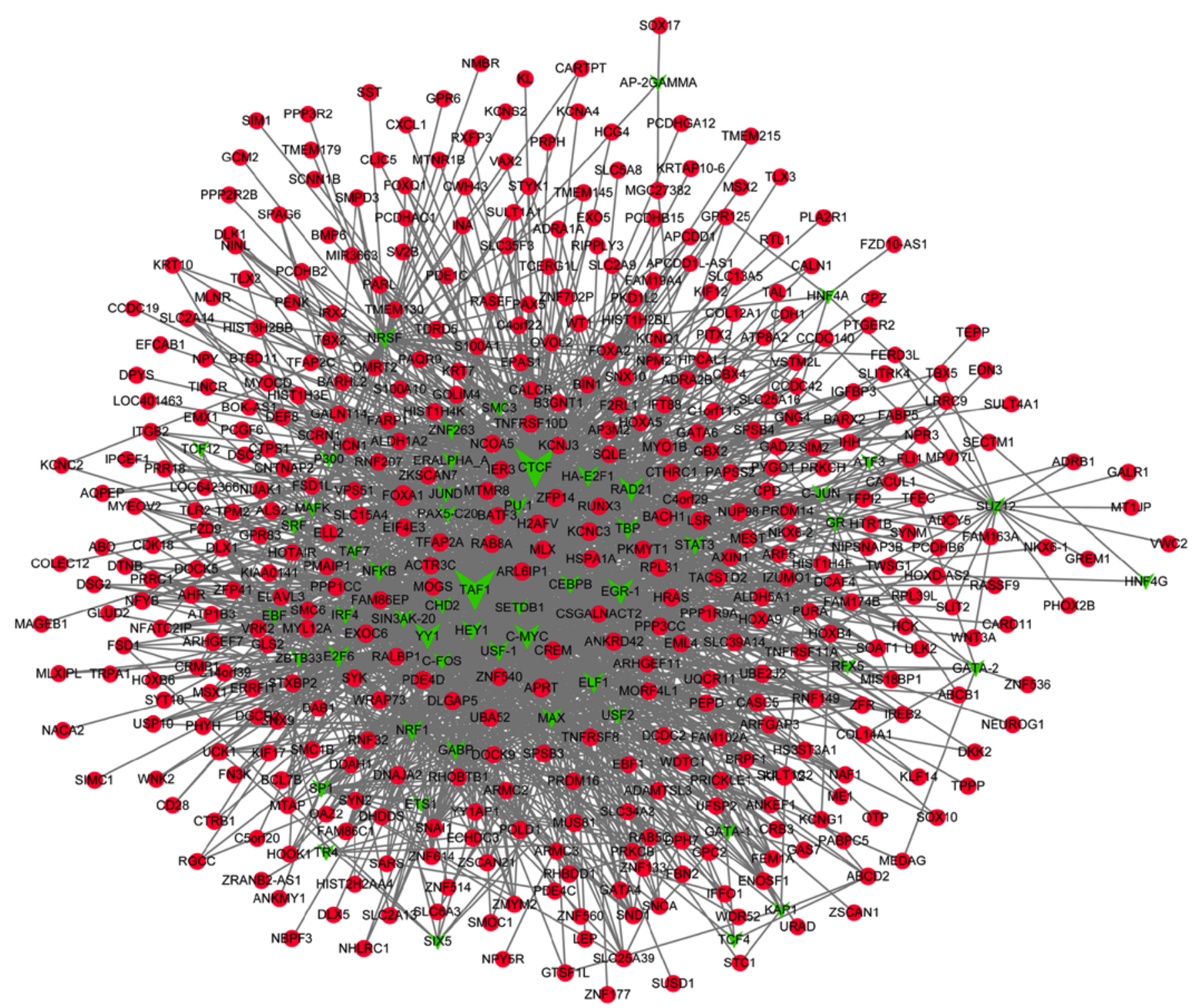

Figure 4. Transcriptional regulatory network of hypermethylated genes. Red nodes represent the hypermethylated gene, green arrows represent transcription factors and the size of node indicates the degree value. Lines/edges represent interactions.

adenine phosphoribosyltransferase (degree, 17) and potassium voltage-gated channel subfamily $\mathrm{C}$ member 3 (KCNC3; degree, 15), and the hypomethylated genes, proliferating cell nuclear antigen-associated factor (degree, 14) and caspase- 8 (CASP8; degree, 12).

Data validation. From the GSE22867 validation dataset, 2,151 DMGs were identified. As demonstrated in Fig. 6, a total of 690 overlapping DMGs were revealed. In particular, the hypermethylated genes $N P Y, F O X A 1$ and $K C N C 3$, as well as the hypomethylated genes $T N F$ and $C A S P 8$, were among the overlapping DMGs.

\section{Discussion}

The current study systemically analyzed the DNA methylation profile of GBM samples to improve our understanding of GBM at the epigenetic level and identify potential biomarkers as therapeutic targets for GBM.
Differential analysis revealed that the methylation levels of 1,326 genes were altered in patients with GBM compared with those in the controls. The biological functions most associated with the DMGs were explored by functional enrichment analysis. The results revealed that DMGs were closely associated with the functions of 'GPCR ligand binding', 'cytokine activity', 'cytokine-cytokine receptor interaction', and 'D-glutamine and D-glutamate metabolism'. Both 'cytokine activity' and 'cytokine-cytokine receptor interaction' are associated with inflammatory responses, and there is a close association between inflammation and cancer. Unresolved inflammation resulting from a failure in the regulation of the immune response creates a tumor microenvironment, an important aspect of tumorigenesis proliferation, survival and migration $(31,32)$. GPCR ligands bind to GPCRs and activate the downstream signaling that regulates cellular physiology. Aberrant G-protein signaling is closely associated with cancer development and progression (33).

To investigate whether abnormally methylated genes are associated with GBM, PPI networks of the screened genes 


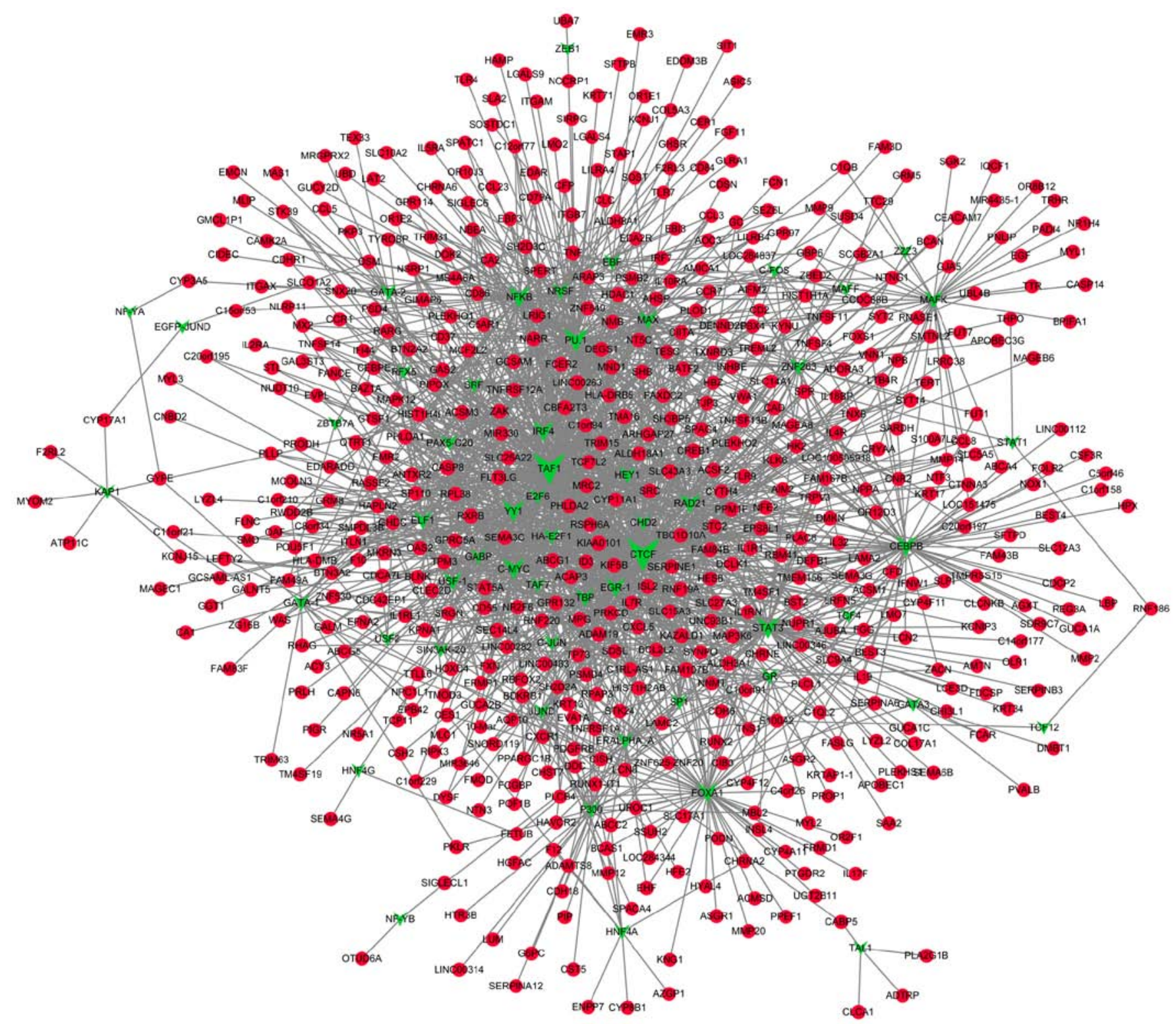

Figure 5. Transcriptional regulatory network of hypomethylated genes. Red nodes represent the hypermethylated gene, green arrows represent transcription factors and the size of node indicates the degree value. Lines/edges represent interactions.

were constructed and the degrees of the nodes were analyzed in the present study. The results demonstrated that the top three genes with the highest node degrees were $A D C Y 2, N P Y$ and $S S T$ in the hypermethylated genes network, and $K N G 1, S R C$ and $T N F$ in the hypomethylated genes network. Among these genes, $N P Y$ and $T N F$ were validated in the alternative dataset. $N P Y$ encodes a 36-amino acid neuropeptide that acts as a neurotransmitter in the brain and autonomic nervous system of humans. Using bioinformatic analysis, abnormal methylation of NPY has been observed in numerous types of cancer (34-36). Furthermore, a previous study reported that $N P Y$ is expressed in various types of intracranial tumor in humans and that $N P Y$ mRNA is detectable in the temporal lobe in higher quantities compared with that in tumors (37). In addition, the present study identified that $T N F$ was hypomethylated, which may lead to upregulation of the gene. The expression of TNF- $\alpha$ has been identified to be increased in GBM (38), which corresponds with the current study. Therefore, NPY and TNF may be involved in GBM due to their abnormal methylation and may result in the disturbance of biological processes.

The hypermethylated genes $F O X A 1$ and $K C N C 3$, as well as the hypomethylated gene $C A S P 8$, exhibited high degrees in the transcriptional regulatory networks, which was also confirmed by the second dataset. FOXAl acts as a TF and has been demonstrated to be a potential regulator of human glioma progression (39). $K C N C 3$, encoding the Kv3. 3 voltage-gated potassium channel, is expressed in various neuronal cell types that are involved in motor function (40). Previous studies have demonstrated an association between $K C N C 3$ expression and the poor prognosis of patients with GBM (41). Patients with GBM with higher $K C N C 3$ expression exhibit improved survival times (42). Genomic loss of CASP8 by DNA methylation may result in tumor resistance to therapies targeting TNF-related apoptosis-inducing ligand-associated apoptosis pathways (43). In summary, the methylation of FOXA1, $K C N C 3$ and $C A S P 8$ in GBM should be investigated in future 
Table IV. Top 10 hypermethylated and hypomethylated genes with high node degree in the transcriptional regulatory networks.

\begin{tabular}{lc}
\hline Gene & Node degree \\
\hline Hypermethylated & \\
FOXA1 & 35 \\
APRT & 17 \\
KCNC3 & 15 \\
LSR & 15 \\
SNAI1 & 13 \\
NUP98 & 13 \\
PDE4D & 12 \\
RPL31 & 12 \\
RPL31 & 12 \\
CREM & 12 \\
Hypomethylated & \\
KIAA0101 & 14 \\
CASP8 & 12 \\
TMA16 & 12 \\
FAM107B & 11 \\
LINCO0263 & 11 \\
RXRB & 11 \\
PHLDA1 & 10 \\
DEGS1 & 11 \\
KAZALD1 & 12 \\
CIRL-AS1 & 10 \\
\hline
\end{tabular}

studies, as this may promote the development of therapeutic approaches.

In conclusion, the current study comprehensively analyzed the DNA methylation profile of GBM using bioinformatics approaches and identified several abnormally methylated genes, including NPY, TNF, FOXA1, KCNC3 and CASP8. The findings of the present study improve the understanding of the molecular mechanism underlying GBM, and provide potential biomarkers for GBM and the development of novel treatment strategies. However, the number of samples included in this study was relatively small. Experimental verification and additional studies with larger sample sizes are required to confirm the present results.

\section{Acknowledgements}

Not applicable.

\section{Funding}

No funding was received.

\section{Availability of data and materials}

The datasets generated and/or analyzed during the current study are available as datasets GSE50923 and GSE22867 from the GEO database (http://www.ncbi.nlm.nih.gov/projects/geo/).

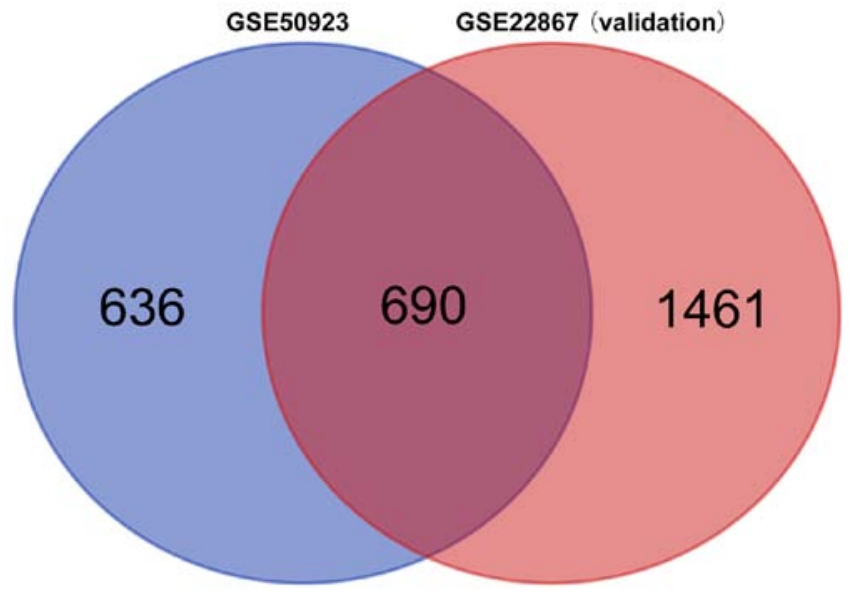

Figure 6. A Venn diagram illustrating data validation using another dataset.

\section{Authors' contributions}

SK and SC conceived and designed the study, and drafted the manuscript. WC acquired, analyzed and interpreted the data, and conducted the statistical analysis. BY acquired and interpreted data, and revised the manuscript for important intellectual content. All authors read and approved the final manuscript.

\section{Ethics approval and consent to participate}

Not applicable.

\section{Patient consent for publication}

Not applicable.

\section{Competing interests}

The authors declare that they have no competing interests.

\section{References}

1. Wen PY and Kesari S: Malignant gliomas in adults. N Engl $\mathbf{J}$ Med 359: 492-507, 2008.

2. Rock K, McArdle O, Forde P, Dunne M, Fitzpatrick D, O'Neill B and Faul C: A clinical review of treatment outcomes in glioblastoma multiforme - the validation in a non-trial population of the results of a randomised phase III: Has a more radical approach improved survival?. Br J Radiol 85: e729-e733 2012.

3. Holland EC: Gliomagenesis: Genetic alterations and mouse models. Nat Rev Genet 2: 120-129, 2001.

4. Nagarajan RP and Costello JF: Epigenetic mechanisms in glioblastoma multiforme. Semin Cancer Biol 19: 188-197, 2009.

5. Herman JG and Baylin SB: Gene silencing in cancer in association with promoter hypermethylation. N Engl J Med 349: 2042-2054, 2003.

6. Karpf AR and Matsui SI: Genetic disruption of cytosine DNA methyltransferase enzymes induces chromosomal instability in human cancer cells. Cancer Res 65: 8635-8639, 2005.

7. Nakamura M, Yonekawa Y, Kleihues P and Ohgaki H: Promoter hypermethylation of the RB1 gene in glioblastomas. Lab Invest 81: 77-82, 2001.

8. Amatya VJ, Naumann U, Weller M and Ohgaki H: TP53 promoter methylation in human gliomas. Acta Neuropathol 110: $178-184,2005$.

9. Baeza N, Weller M, Yonekawa Y, Kleihues P and Ohgaki H: PTEN methylation and expression in glioblastomas. Acta Neuropathol 106: 479-485, 2003. 
10. Kreth $\mathrm{S}$, Thon $\mathrm{N}$, Eigenbrod $\mathrm{S}$, Lutz J, Ledderose $\mathrm{C}$ Egensperger R, Tonn JC, Kretzschmar HA, Hinske LC and Kreth FW: O-methylguanine-DNA methyltransferase (MGMT) mRNA expression predicts outcome in malignant glioma independent of MGMT promoter methylation. PLoS One 6: e17156, 2011.

11. Lalezari S, Chou AP, Tran A, Solis OE, Khanlou N, Chen W, Li S, Carrillo JA, Chowdhury R, Selfridge J, et al: Combined analysis of O6-methylguanine-DNA methyltransferase protein expression and promoter methylation provides optimized prognostication of glioblastoma outcome. Neuro Oncol 15: 370-381, 2013.

12. Garcia-Manero G: Demethylating agents in myeloid malignancies. Curr Opin Oncol 20: 705-710, 2008.

13. Li D, Xie Z, Pape ML and Dye T: An evaluation of statistical methods for DNA methylation microarray data analysis. BMC Bioinformatics 16: 217, 2015.

14. Xu C, Zhang JG, Lin D, Zhang L, Shen H and Deng HW: A systemic analysis of transcriptomic and epigenomic data to reveal regulation patterns for complex disease. G3 (Bethesda) 7: 2271-2279, 2017.

15. Smith AA, Huang YT, Eliot M, Houseman EA, Marsit CJ, Wiencke JK and Kelsey KT: A novel approach to the discovery of survival biomarkers in glioblastoma using a joint analysis of DNA methylation and gene expression. Epigenetics 9: 873-883, 2014.

16. Gadaleta F, Bessonov K and Van Steen K: Integration of gene expression and methylation to unravel biological networks in glioblastoma patients. Genet Epidemiol 41: 136-144, 2017.

17. Lai RK, Chen Y, Guan X, Nousome D, Sharma C, Canoll P, Bruce J, Sloan AE, Cortes E, Vonsattel JP, et al: Genome-wide methylation analyses in glioblastoma multiforme. PLoS One 9: e89376, 2014.

18. Du P, Kibbe WA and Lin SM: Lumi: A pipeline for processing Illumina microarray. Bioinformatics 24: 1547-1548, 2008.

19. Du P, Zhang X, Huang CC, Jafari N, Kibbe WA, Hou L and Lin SM: Comparison of Beta-value and M-value methods for quantifying methylation levels by microarray analysis. BMC Bioinformatics 11: 587, 2010.

20. Du P and Bourgon R: methyAnalysis: An R package for DNA methylation data analysis and visualization, 2013.

21. Harris MA, Clark J, Ireland A, Lomax J, Ashburner M, Foulger R, Eilbeck K, Lewis S, Marshall B, Mungall C, et al: The gene ontology (GO) database and informatics resource. Nucleic Acids Res 32: D258-D261, 2004.

22. Kanehisa M and Goto S: KEGG: Kyoto encyclopedia of genes and genomes. Nucleic Acids Res 28: 27-30, 2000.

23. Joshi-Tope G, Gillespie M, Vastrik I, D'Eustachio P, Schmidt E, de Bono B, Jassal B, Gopinath GR, Wu GR, Matthews L, et al: Reactome: A knowledgebase of biological pathways. Nucleic Acids Res 33: D428-D432, 2005.

24. Shannon P, Markiel A, Ozier O, Baliga NS, Wang JT, Ramage D, Amin N, Schwikowski B and Ideker T: Cytoscape: A software environment for integrated models of biomolecular interaction networks. Genome Res 13: 2498-2504, 2003.

25. Zhao M, Sun J and Zhao Z: TSGene: A web resource for tumor suppressor genes. Nucleic Acids Res 41: D970-D976, 2013.

26. Chen JS, Hung WS, Chan HH, Tsai SJ and Sun HS: In silico identification of oncogenic potential of fyn-related kinase in hepatocellular carcinoma. Bioinformatics 29: 420-427, 2013.

27. ENCODE Project Consortium: The ENCODE (ENCyclopedia of DNA elements) project. Science 306: 636-640, 2004.

28. Landt SG, Marinov GK, Kundaje A, Kheradpour P, Pauli F, Batzoglou S, Bernstein BE, Bickel P, Brown JB, Cayting P, et al: ChIP-seq guidelines and practices of the ENCODE and modENCODE consortia. Genome Res 22: 1813-1831, 2012.
29. Etcheverry A, Aubry M, de Tayrac M, Vauleon E, Boniface R, Guenot F, Saikali S, Hamlat A, Riffaud L, Menei P, et al: DNA methylation in glioblastoma: Impact on gene expression and clinical outcome. BMC Genomics 11: 701, 2010.

30. Gentleman R, Carey V, Huber W and Hahne F: Genefilter: Methods for filtering genes from microarray experiments. R Package version 1.66.0, 2011.

31. Eiró $\mathrm{N}$ and Vizoso FJ: Inflammation and cancer. World $\mathrm{J}$ Gastrointest Surg 4: 62-72, 2012.

32. Coussens LM and Werb Z: Inflammation and cancer. Nature 420: 860-867, 2002

33. Spiegelberg BD and Hamm HE: Roles of G-protein-coupled receptor signaling in cancer biology and gene transcription. Curr Opin Genet Dev 17: 40-44, 2007.

34. Anglim PP, Alonzo TA and Laird-Offringa IA: DNA methylation-based biomarkers for early detection of non-small cell lung cancer: An update. Mol Cancer 7: 81, 2008.

35. Hill VK, Ricketts C, Bieche I, Vacher S, Gentle D, Lewis C, Maher ER and Latif F: Genome-wide DNA methylation profiling of $\mathrm{CpG}$ islands in breast cancer identifies novel genes associated with tumorigenicity. Cancer Res 71: 2988-2999, 2011.

36. Shin SH, Kim BH, Jang JJ, Suh KS and Kang GH: Identification of novel methylation markers in hepatocellular carcinoma using a methylation array. J Korean Med Sci 25: 1152-1159, 2010.

37. Knerr I, Schuster S, Nomikos P, Buchfelder M, Dötsch J, Schoof E, Fahlbusch R and Rascher W: Gene expression of adrenomedullin, leptin, their receptors and neuropeptide $\mathrm{Y}$ in hormone-secreting and non-functioning pituitary adenomas, meningiomas and malignant intracranial tumours in humans. Neuropathol Appl Neurobiol 27: 215-222, 2001.

38. Kore RA and Abraham EC: Inflammatory cytokines, interleukin-1 beta and tumor necrosis factor-alpha, upregulated in glioblastoma multiforme, raise the levels of CRYAB in exosomes secreted by U373 glioma cells. Biochem Biophys Res Commun 453: 326-331, 2014.

39. Wang L, Qin H, Li L, Feng F, Ji P, Zhang J, Li G, Zhao Z and Gao G: Forkhead-box A1 transcription factor is a novel adverse prognosis marker in human glioma. J Clin Neurosci 20: 654-658, 2013.

40. Hurlock EC, McMahon A and Joho RH: Purkinje-cell-restricted restoration of Kv3.3 function restores complex spikes and rescues motor coordination in Kenc3 mutants. J Neurosci 28: 4640-4648, 2008.

41. Martinez R, Stuhmer W, Martin S, Schell J, Reichmann A, Rohde V and Pardo L: Analysis of the expression of Kv10.1 potassium channel in patients with brain metastases and glioblastoma multiforme: Impact on survival. BMC Cancer 15: 839, 2015.

42. Arvind S, Arivazhagan A, Santosh V and Chandramouli BA: Differential expression of a novel voltage gated potassium channel-Kv 1.5 in astrocytomas and its impact on prognosis in glioblastoma. Br J Neurosurg 26: 16-20, 2012.

43. Qi L, Bellail AC, Rossi MR, Zhang Z, Pang H, Hunter S, Cohen C, Moreno CS, Olson JJ, Li S and Hao C: Heterogeneity of primary glioblastoma cells in the expression of caspase- 8 and the response to TRAIL-induced apoptosis. Apoptosis 16: 1150-1164, 2011.

This work is licensed under a Creative Commons Attribution-NonCommercial-NoDerivatives 4.0 International (CC BY-NC-ND 4.0) License. 